\title{
Atractividad - Competitividad de destino turístico. Evaluación de Holguín para el mercado estadounidense
}

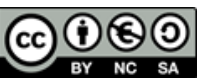

\section{Attractiveness - Tourism destination competitiveness. Evaluation of Holguín for US market}

MSc. Justa Ramona Medina Labrada. ${ }^{1}$, Lic. Elizabeth del Carmen Pérez Ricardo. ${ }^{2}$ \& Lic. Milagros de las Mercedes Riquenes Gainza. ${ }^{3}$

Recibido: 13-09-2019 / Revisado: 14-09-2019 / Aceptado: 17-09-2019 / Publicado: 05-10-2019

Resumen. $\quad$ DOI: $\underline{\text { https://doi.org/10.33262/concienciadigital.v2i4.851 }}$

El restablecimiento de las relaciones diplomáticas entre Cuba y Estados Unidos en 2015 provocó un crecimiento importante del flujo de visitantes estadounidenses a Cuba. A pesar de las restricciones legales que en la actualidad desestimulan dichos flujos, se consideró conveniente evaluar la atractividad - competitividad del destino turístico Holguín para distintos segmentos de clientes estadounidenses. Para desarrollar el estudio se propuso un procedimiento, que considera las mejores experiencias precedentes y que combina el análisis de la atractividad y la competitividad. Como resultado de la aplicación del procedimiento se determinaron los aspectos favorables y desfavorables que presenta el destino Holguín con relación a la atractividad turística para cada segmento estudiado; así como la posición competitiva que ocupa Holguín para los segmentos de yatistas, cruceristas y los que acceden por vía aérea. Se realizaron recomendaciones para favorecer la gestión efectiva del destino Holguín para dicho mercado.

Palabras claves: atractividad, competitividad, destino turístico, segmentos de mercado estadounidenses

\footnotetext{
${ }^{1}$ Universidad de Holguín. Ministerio de Educación Superior. Cuba., jmedina@uho.edu.cu

${ }^{2}$ Universidad de Holguín. Ministerio de Educación Superior. Cuba., elizabeth.ricardo@uho.edu.cu

${ }^{3}$ ISLAZUL Holguín. Ministerio de Turismo., milir@ nauta.cu
} 


\section{Abstract.}

The restoration of diplomatic relations between Cuba and the United States in 2015 caused a significant growth in the flow of American visitors to Cuba. In spite of the legal restrictions currently discouraging such flows, it was considered convenient to evaluate the attractiveness - competitiveness of the Holguín tourist destination for different segments of US clients. To develop the study, a procedure was proposed, which considers the best previous experiences and combines the analysis of attractiveness and competitiveness. As a result of the application of the procedure were determined the favorable and unfavorable aspects of the Holguín destination in relation to the tourist attractiveness for each segment studied; as well as the competitive position occupied by Holguin for the segments of yachtsmen, cruise passengers and those who access by air. Recommendations were made to favor the effective management of the Holguín destination for this market.

Keywords: Attractiveness, competitiveness, tourist destination, US market segments.

\section{Introducción.}

Durante las seis últimas décadas, el turismo ha experimentado una continua expansión y diversificación, convirtiéndose en uno de los sectores económicos de mayor envergadura y crecimiento del mundo. Un número creciente de destinos se han abierto al turismo y han invertido en él, haciendo del mismo un sector clave para el progreso socioeconómico, a través de la creación de puestos de trabajo y de empresas, la generación de ingresos de exportación y la ejecución de infraestructuras [1].

En este contexto el análisis de la atractividad de destinos turísticos es fundamental, dado que permite contar con una herramienta esencial para la elección de negocios turísticos que se consideren más atractivos y para los que se tengan mejores condiciones para competir, además permite dar solución a un problema fundamental en la inadecuada forma de gestión de los negocios turísticos: el modo erróneo de penetrar en la competencia turística [2]. Un destino es competitivo si puede atraer y satisfacer a turistas potenciales y que esta competitividad se determina tanto por los factores específicos del turismo, como por un abanico más amplio de factores que influyen a los proveedores del servicio turístico [3].

Si se considera que el restablecimiento de las relaciones diplomáticas con Estados Unidos en 2015y la flexibilizaron los viajes de estadounidenses tanto por vía aérea como marítima implicaron un crecimiento sustancial del arribo de estadounidenses a Cuba de un 76,66 por ciento en 2016 y un 117,56 por ciento en 2017, unido a la importancia de evaluar la atractividad y competitividad en los destinos se consideró conveniente profundizar y aplicar estos estudios en el destino Holguín para el mercado estadounidense [4; 5].

El mayor volumen de arribos se produce por vía aérea, favorecido por el establecimiento de numerosos vuelos regulares a varios destinos cubanos, entre ellos Holguín. También se han 
dinamizado los segmentos de yatistas y cruceristas en Cuba, que se ubica en una de las regiones principales a nivel mundial para el desarrollo de la actividad náutica. Se estima que entre las costas del sur de Estados Unidos y Cuba navegan más de un millón de yates y que una vez que se levanten las restricciones de viajes impuestas por el gobierno de los Estados Unidos un aproximado de 60000 yates estadounidenses atracarán en puerto cubano. En este escenario se ha incrementado progresivamente el arribo de yatistas a distintas marinas del país y Holguín posee la Marina Internacional de Vita, con facilidades para el atraque de yates, además de atractivos de interés para este segmento [6; 7].

Por otra parte, se realizan operaciones de cruceros estadounidenses en los puertos de La Habana, Cienfuegos y Santiago de Cuba. Holguín, por su posición geográfica, sus potencialidades en relación con la oferta turística actual y perspectiva y las operaciones de cruceros europeos realizadas por el puerto de Antilla, tiene posibilidades de incluirse en los circuitos de cruceros estadounidenses.

A todo ello se une que desde la propia actualización del modelo de desarrollo económico cubano aprobada en el VI Congreso del Partido Comunista de Cuba se definió como una meta para el sector turístico "crear, diversificar y consolidar de forma acelerada servicios y ofertas complementarias al alojamiento que distingan al país, priorizando el desarrollo de las modalidades: ... marinas y náutica..." (Lineamiento 260). Esta política fue ratificada en el VII Congreso del PCC, donde se plantea en el lineamiento 208 "Continuar incrementando la competitividad de Cuba en los mercados turísticos, diversificando las ofertas...” [8; 9].

A pesar de las restricciones legales que en la actualidad desestimulan los flujos turísticos de estadounidenses a Cuba, se consideró conveniente evaluar la atractividad - competitividad del destino turístico Holguín para segmentos de clientes estadounidense, lo que constituye el objetivo de la presente investigación. Ello podrá favorecer la elección o ampliación de los negocios turísticos con mayores posibilidades de éxito en este destino, una vez que se flexibilicen las restricciones a los estadounidenses para viajar a Cuba.

\section{Desarrollo.}

Existen distintos métodos para medir la competitividad, entre ellos se destacan: Modelo de las fuerzas competitivas de Porter, Modelo de Calgary de Crouch y Ritchie y Modelo integrado de Dwyer y Kim. Este último es la obra más importante en el análisis de la competitividad en el turismo. Los modelos anteriormente referidos, basan su funcionamiento en medir únicamente la competitividad en sus diferentes modalidades y excluyen el análisis de atractividad en ellos. Por otra parte, muchos de los factores identificados como cruciales de la competitividad no se pueden aplicar por la falta de indicadores disponibles y no comparables entre los diferentes destinos, por lo que a todos se les señala la gran complejidad que poseen a partir de la excesiva cantidad de indicadores y las dificultades y altos costos para obtener la información $[10 ; 11 ; 12 ; 13]$. 
Por estas razones fue necesario proponer un procedimiento más sencillo y ajustado a los intereses de la presente investigación, que partió de las experiencias precedentes y que favorece la obtención de la información necesaria sobre las variables a considerar para el estudio, por los propios investigadores. En la figura 1 se expresa gráficamente el procedimiento propuesto y seguidamente se explican de forma resumida los objetivos, descripción y técnicas a utilizar en las diferentes etapas y pasos.

Figura N 1. Procedimiento para diagnosticar la atractividad competitividad de un destino turístico para un mercado.

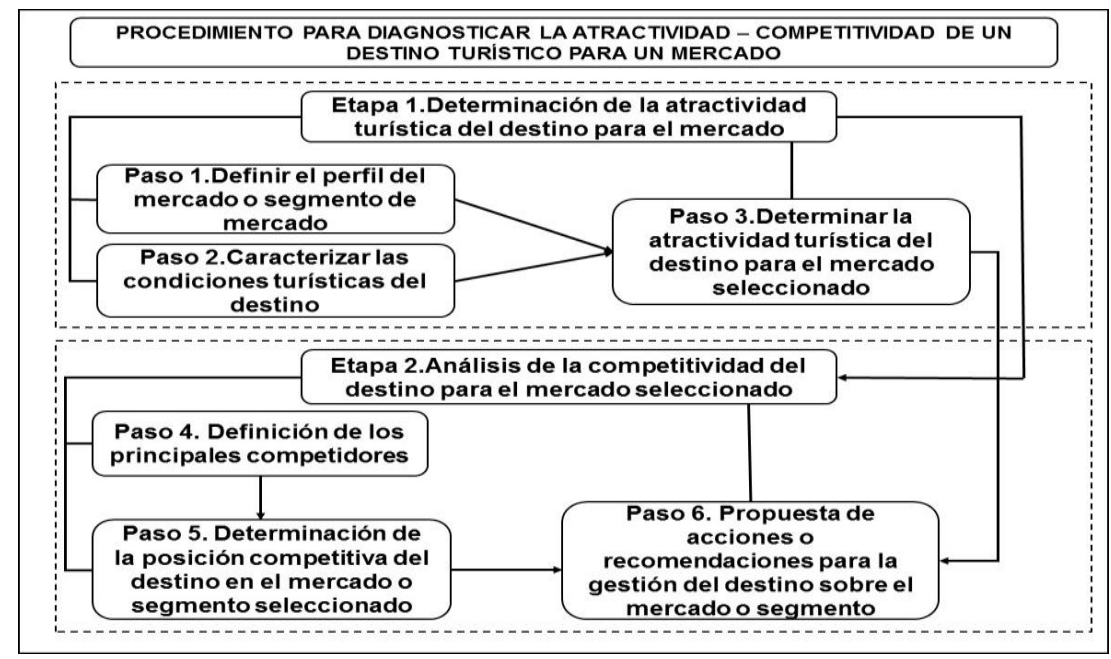

Fuente: Elaboración propia.

Objetivo: Determinar la atractividad-competitividad turística de un destino para un segmento de mercado.

Etapa 1: Determinación de la atractividad turística del destino para el mercado o segmento seleccionado

Objetivo: Evaluar la correspondencia existente entre las condiciones turísticas del destino y las preferencias y características del mercado, precisando los aspectos favorables y desfavorables.

Paso1: Definir el perfil del mercado o segmento de mercado

Descripción: A partir de un conjunto de variables determinadas se debe conformar el perfil del mercado o segmento en estudio con la mayor amplitud y detalle posible.

Técnicas a emplear: Búsqueda de información secundaria en documentos de organizaciones territoriales, nacionales, internacionales y otras publicaciones; así como entrevistas.

Paso 2: Caracterizar las condiciones turísticas del destino

Descripción: Para caracterizar las condiciones del destino para el desarrollo de un mercado o segmento se proponen un conjunto de variables a caracterizar, a partir de las dimensiones y variables de atractividad [14]. 
Técnicas a emplear: Entrevistas, revisión de informes de organizaciones turísticas del destino y búsqueda de información secundaria en Internet

Paso 3: Determinar la atractividad turística del destino para el mercado seleccionado Descripción: Se deben identificar los Factores Clave de Éxito (FCE), a partir de alinear los aspectos más importantes para los clientes, contemplados en la caracterización del mercado, con la visión del destino y compararlos con las condiciones turísticas del destino, definidas en el paso dos, determinando los aspectos favorables y desfavorables en relación con la atractividad.

Técnicas a emplear: Estudio de caso para determinar los FCE y generación de ideas y técnicas de consenso para determinar los aspectos favorables y desfavorables

Etapa 2: Análisis de la competitividad del destino para el mercado seleccionado

Objetivo: Determinar la posición que ocupa el destino en relación con los competidores para el mercado o segmento seleccionado y proponer acciones o recomendaciones para la gestión del destino sobre el mercado o segmento seleccionado.

Paso 4: Definición de los principales competidores

Descripción: Se deben definir los principales competidores de la organización en el área geográfica determinada, teniendo en cuenta los que mayor importancia o cuota de mercado.

Técnicas a emplear: Estudio de caso y búsqueda de información secundaria en Internet

Paso 5: Determinar la posición competitiva del destino en el mercado o segmento seleccionado

Descripción: Se deben evaluar los resultados de la gestión del destino en dicho mercado o segmento y su comparación con los resultados de los competidores, también en relación con el mercado o segmento seleccionado y determinar la posición competitiva del destino respecto a los competidores, considerando un grupo de 13 indicadores que fueron definidos a partir del estudio de los indicadores más empleados por los autores que abordan este tema, buscando utilizar los más valiosos para realizar el análisis y a la vez que sea factible la obtención y comparación de la información.

Como quiera que se consideran indicadores cuantitativos y cualitativos, se requiere homogeneizar los datos obtenidos, para lo cual se debe utilizar la fórmula de estandarización [15].

$$
Z_{i}=\frac{X_{i}-X_{i} \operatorname{mín}}{X_{i} m a ́ x-X_{i} \min }
$$

donde:

Zi: Valor estandarizado

Xi: valor de la variable

Xi mín: mínimo valor de las variables

Xi máx: máximo valor de las variables 
Luego se integran los datos en un índice, para lo cual se propone el método del promedio simple, donde se suman los factores y se dividen entre el número total de factores analizados. Este método simplifica la complejidad del cálculo y permite el objetivo que se persigue, la integración de los factores en un índice, que permite determinar la posición competitiva de los destinos evaluados.

Técnicas a emplear: Análisis documental, búsqueda de información secundaria en Internet, entrevistas, criterio de especialistas, trabajo en grupos, procesador de datos Microsoft Office Excell y estadística descriptiva

Paso 6: Propuesta de acciones o recomendaciones para la gestión del destino sobre el mercado o segmento

Descripción: Se deben proponer acciones o recomendaciones para la gestión del destino sobre el mercado o segmento seleccionado, a partir de los elementos de atractividad desfavorables definidos en el paso 3 , de la posición competitiva que ocupa el destino determinada en el paso 5 y de la experiencia obtenida por el investigador durante el desarrollo del estudio, que sirvan de guía u orientación para la toma de decisiones.

Técnicas a emplear: Estudio de caso y generación de ideas

\section{Resultados.}

La aplicación de este procedimiento para el destino turístico Holguín dirigido a los segmentos de clientes estadounidenses que arriban por vía marítima (yatistas y cruceristas) y aérea permitió obtener como principales resultados los siguientes.

\section{Para el segmento de yatistas estadounidenses.}

Para realizar el análisis de la atractividad se identificaron los aspectos más importantes para los yatistas estadounidenses, que se alinean con la visión del destino; los que se definieron como Factores Clave de Éxito (FCE) y luego se compararon, a través de estudio de casos, con las condiciones turísticas de Holguín, lo que permitió determinar los aspectos favorables y también los aspectos desfavorables de Holguín para los yatistas estadounidenses, los que se detallan a continuación.

Aspectos favorables

1. Existencia de numerosos atractivos culturales y naturales de interés para el segmento.

2. La Marina Internacional de Vita posee servicios básicos para el yatista que favorecen la estancia de los mismos en sus instalaciones.

3. El destino Holguín cuenta con amplios programas de actividades para satisfacer las necesidades de las parejas, familias, grupos de amigos y otras.

4. El destino cuenta con una oferta culinaria autóctona, especialmente en Gibara, además la Marina Internacional de Vita incluye la restauración como un servicio complementario al yatista. 
5. Presencia de alojamiento estatal y no estatal con las características y estándares de calidad requeridos para satisfacer el segmento de yatistas estadounidenses.

6. La práctica adecuada del idioma inglés por parte de los trabajadores del sector turístico y de la población en general.

7. En el destino se ofrecen diversas actividades náuticas, como la pesca, buceo contemplativo, paseos en catamarán, etc., además de los servicios en los puntos náuticos.

\section{Aspectos desfavorables}

1. Insuficiente presencia de información sobre las facilidades de la Marina Internacional de Vita y del destino Holguín en Internet y redes sociales.

2. Insuficiencias de la red de venta y otros servicios en la marina Puerto de Vita para asegurar todo el aprovisionamiento requerido por los yatistas.

3. Aplicación en Cuba del gravamen del 10 por ciento del cambio de moneda del dólar estadounidense al peso convertible cubano.

Para determinar la posición competitiva del destino turístico Holguín para el segmento de yatistas estadounidenses se debe considerar que Holguín es un mercado potencial para los yatistas procedentes de Estados Unidos, por lo que los indicadores cuantitativos incluidos en el procedimiento no se consideran en el análisis, ya que no serían comparables con los destinos que si operan ya con dicho segmento. El análisis se realizó con siete de las variables propuestas en el procedimiento, caracterizando cada una en los diferentes destinos. Como quiera que las variables utilizadas son cualitativas se le otorgó a cada una un valor cuantitativo, a partir de la caracterización realizada, utilizando una escala donde 5 equivale a muy favorable, 4 a favorable, 3 a medianamente favorable, 2 desfavorable y 1 equivale a muy desfavorable. Para otorgar la calificación anterior a las variables por destino se utilizó el criterio de especialistas. Luego se estandarizaron los resultados y se calculó un índice de competitividad para cada destino que integra la evaluación de las variables consideradas.

Las variables consideradas para este segmento fueron: Recursos turísticos, reconocimiento de preparación y profesionalidad del capital humano, seguridad y tranquilidad en el destino, gestión medio - ambiental, aceptación de los residentes, infraestructura de servicio y facilidades de transporte interno. Una vez procesada la información se determinó un índice de competitividad del destino Holguín de 0,2143. Así mismo Cienfuegos alcanzó un índice de 0,1714, Varadero 0,2857 y La Habana 0, 2381.

\section{Segmento de cruceristas estadounidenses}

Se siguieron los pasos previstos en el procedimiento y descritos anteriormente y se determinaron los aspectos favorables y desfavorables del destino Holguín para este segmento, los que se detallan a continuación.

\section{Favorables}

1. Cercanía entre el país emisor y receptor.

2. Existencia de gran número de atractivos tanto naturales como culturales, que se encuentran entre las preferencias de los cruceristas, destacándose las ofertas 
culturales y recreativas de las ciudades de Antilla, Banes y Holguín, así como la presencia de otros atractivos importantes cercanos al puerto de Antilla.

3. La población local posee un alto nivel de implicación con la actividad turística.

4. Excelentes condiciones climáticas en el destino para el desarrollo de esta modalidad, por la escasa presencia de eventos naturales que la puedan interferir.

5. Buen nivel de idioma por parte de los trabajadores del sector y de los residentes Entre los aspectos desfavorables se encuentran:

1. Poco acondicionamiento del área de atraque en el puerto de Antilla.

2. Insuficiencia de la red de compras en el destino.

3. Desventajas en las tasas de cambio entre el dólar estadounidense y el CUC, al aplicársele un gravamen del 10 por ciento.

4. Dificultades para acceder a las ofertas extra hoteleras por Internet desde cualquier punto y también desde los teléfonos inteligentes.

Seguidamente se calculó el índice de competitividad considerando los destinos cubanos de cruceros en la actualidad. Las variables consideradas para este segmento fueron: Recursos turísticos, preparación y profesionalidad del capital humano, seguridad y tranquilidad en el destino, gestión medio - ambiental, aceptación de los residentes, infraestructura de servicio y facilidades de transporte interno. Una vez procesada la información se determinó un índice de competitividad que arrojó como resultado que Holguín posee un indicador de 0,0714, La Habana o,2857, Cienfuegos 0, 0952 y Santiago de Cuba 0,2381.

\section{Clientes que acceden por vía aérea}

La aplicación de los pasos previstos en el procedimiento para el segmento que accede al destino por vía aérea permitió determinar los aspectos favorables y desfavorables con que cuenta el destino Holguín para atraer flujos turísticos del mercado estadounidense. Los mismos se relacionan a continuación.

\section{Aspectos favorables}

1. Existe vinculación aérea directa entre el país emisor y el receptor, a través de numerosos vuelos regulares directos.

2. Holguín posee atractivos histórico-culturales como monumentos, museos, sitios patrimoniales, productos vinculados a la naturaleza y para la recreación diurna y nocturna; así como facilidades náuticas que permiten satisfacer sus principales motivos de viaje y desarrollar las actividades turísticas que prefieren.

3. Existen en el destino hoteles con las características y estándares de calidad que prefieren los turistas estadounidenses, los cuales están preparados para atender clientes con un promedio de días de estancia igual o superior al de este mercado y ofrecen amplios programas de actividades para satisfacer las necesidades de los viajeros solitarios. Así mismo cuenta con casas de renta de buen estándar en varias ciudades y pueblos para los que prefieren este tipo de alojamiento

4. La preparación en idioma inglés de los trabajadores del sector del turismo en el destino Holguín es buena 


\section{Aspectos desfavorables}

1. La red de tiendas y centros comerciales del destino Holguín es insuficiente para satisfacer una de sus actividades preferidas: las compras

2. Insuficientes facilidades para satisfacer las necesidades de búsqueda de información a través de los teléfonos inteligentes

3. El cambio de moneda se efectúa aplicando un gravamen del $10 \%$ lo que actúa en sentido contrario a la motivación de los clientes

Luego se realizó la evaluación de la competitividad considerando los principales destinos del Caribe. Las variables consideradas fueron: Nivel de utilización de la tecnología para la información y las comunicaciones en la promoción y distribución de servicios y ofertas, preparación y profesionalidad del capital humano, condiciones de seguridad y tranquilidad, gestión medio ambiental, disponibilidad de la infraestructura necesaria y facilidades de transporte dentro del destino. Se obtuvo como resultado que Holguín alcanzó un índice de 0,4285; La Habana 0, 5714; Cancún 0,5714 y Montego Bay 0,4285.

\section{Discusión}

La evaluación y resultados obtenidos en cada segmento de mercado permitieron realizar un conjunto de recomendaciones para favorecer el incremento de la competitividad turística del destino Holguín y una gestión más eficaz.

Para el segmento de yatistas estadounidenses Holguín ocupa el tercer puesto dentro de los destinos analizados, ubicándose por encima de Cienfuegos y por debajo de Matanzas y La Habana con incidencia negativa de la infraestructura del Puerto de Vita y resaltando positivamente el reconocimiento de los clientes sobre la preparación y profesionalidad de los recursos humanos. Se propusieron como principales acciones o recomendaciones que se detallan seguidamente.

1. Incrementar la comunicación promocional de la Marina Internacional de Vita a través de los sitios de los turoperadores que trabajan este segmento.

2. Ampliar la información sobre las facilidades de la Marina Internacional de Vita y los atractivos del destino Holguín a través de las redes sociales, principalmente: active captain, Facebook y el sitio tripadvisor.

3. Realizar acciones de relaciones públicas online con clubes de yatistas estadounidense.

4. Ampliar la infraestructura de servicios en la Marina Internacional de Vita que incluya: Tiendas, diversos restaurantes, facilidades de acceso a Internet, reserva de excursiones, entre otros servicios; así como completar la infraestructura de apoyo para satisfacer todas las necesidades de aprovisionamiento del yatista.

Para el segmento de cruceristas el destino Holguín se encuentra posicionado en el último lugar dentro de los competidores evaluados. Las acciones y recomendaciones propuestas se resumen en:

1. Profundizar en los estudios de factibilidad sobre cuál debe ser el puerto de cruceros de Holguín. 
2. Completar la infraestructura necesaria en el puerto de Antilla para la operación de cruceros (muelle, dragado, comunicaciones, información turística, facilidades para el cambio de moneda, etc.).

3. Ofertar una amplia variedad de excursiones a los cruceristas, fundamentalmente vinculadas a las ciudades, en correspondencia con los intereses de los clientes

4. Recomendar a la dirección del destino y a las organizaciones transportistas de turismo, MITRANS y sector no estatal dar las facilidades para satisfacer todas las necesidades de traslado interno.

5. Estudiar y ejecutar las soluciones necesarias para que los cruceristas realicen las compras en todos los lugares a visitar, incluyendo el puerto de atraque.

Para el segmento que accede por vía aérea el destino turístico Holguín se encuentra al mismo nivel de Montego Bay y por debajo de La Habana y Cancún. Los aspectos que más inciden negativamente en este resultado son el nivel de utilización de la tecnología y las facilidades de transporte dentro del destino. Para mejorar la posición competitiva de Holguín se propusieron las siguientes acciones o recomendaciones:

1. Ampliar la disponibilidad de autos para la renta y el transporte turístico público en el destino.

2. Aprovechar las tecnologías de la información y las comunicaciones disponibles en función de la promoción y comercialización del destino.

3. Crear condiciones tecnológicas para satisfacer la demanda de información y reserva de los clientes desde sus teléfonos inteligentes.

4. Ampliar la red de tiendas y centros comerciales del destino, así como la variedad de sus surtidos

El análisis realizado permitió concluir que en sentido general son más y de mayor peso los aspectos favorables que las insuficiencias, destacándose la capacidad del destino para satisfacer la mayoría de las preferencias de los turistas estadounidenses, en particular los atractivos con que cuenta y la posibilidad de solucionar en el corto y mediano plazo varios de los aspectos desfavorables.

\section{Conclusiones.}

- La complejidad de los modelos para medir la competitividad de destinos turísticos consultados y la no integración en ellos de variables que consideren también la atractividad, implicaron la necesidad de proponer un procedimiento más sencillo y ajustado a los intereses de la presente investigación, que solucionara además la inclusión del análisis de la atractividad en el mismo y favoreciera la obtención de la información por parte de los investigadores.

- Se determinó que el destino turístico Holguín es atractivo para los segmentos de clientes estadounidenses que arriban por vía aérea y marítima (yatistas y cruceristas), 
resaltando como aspectos más importantes y comunes para los tres segmentos los siguientes:

- La diversidad de atractivos culturales y naturales que posee.

- Disponibilidad en el destino de alojamiento estatal y no estatal adecuado.

- Cercanía entre el país emisor y receptor.

- Existencia de acceso aéreo directo y facilidades para el acceso marítimo.

- Buen nivel de idioma por parte de los trabajadores del sector y de los residentes.

- La población local posee un nivel alto de implicación con la actividad turística.

- Se determinaron como desventajas más importantes, en cuanto a la atractividad, las siguientes:

- La red de tiendas y centros comerciales del destino Holguín es insuficiente.

- Aplicación en Cuba del gravamen del 10 por ciento del cambio de moneda del dólar estadounidense al peso convertible cubano.

- Insuficiente comunicación promocional de los atractivos y facilidades del destino Holguín y pocas facilidades para satisfacer las necesidades de búsqueda de información y reserva a través de Internet y los teléfonos inteligentes.

- Necesidad de completar la infraestructura marítima, en especial para el atraque de cruceros.

- Se determinó la posición competitiva del destino turístico Holguín respecto a los competidores en cada uno de los segmentos, precisándose que en general la posición es desventajosa, pero con potencialidades para mejorar los resultados en el corto y mediano plazo.

\section{Referencias bibliográficas.}

OMT (2015). Panorama OMT 2015

Magallanes, A.J. (2004). “Atractividad de mercados turísticos”. Internacional Thomson Editores. México.

Enright. J. M \& Newton, J. (2004) “Tourism destination competitiveness: a quantitative approach”. Tourism Management (25).

ONEI (2017). Anuario Estadístico de Cuba. Disponible en www.onei.cu

ONEI (2018). Anuario Estadístico de Cuba. Disponible en www.onei.cu 
Gómez González, A. (2015). Hello Cuba. Recuperado de http://oncubamagazine.com/economia-negocios/hello-cuba/. Consultado en abril de 2016

Díaz Escrich, J. M (2015). Aseguran que más de 60 mil yates estadounidenses podrían visitar Cuba anualmente. Publicación electrónica Cubadebate

PCC (2016). Lineamientos de la Política Económica y Social del Partido y la Revolución PCC (2016). Actualización de los Lineamientos de la Política Económica y Social del Partido y la Revolución para el período 2016 - 2021

Porter, M. (1984). "Estrategia competitiva”. CECSA, México

Crouch, B. \&,Ritchie I. (2003). "Attractivité de la France: analyse, perception et mesure. Économie et Statistiques"

Dwyer, L. y Kim, CH. (2003). Destination Competitiveness: Determinants and indicators

Hong, H. \& Kacperczyk, M. (2009). "The Price of sin: The effects of social norms on markets". $\quad$ EL $\quad$ SEVIER. Recuperado de http://pages.stern.nyu.edu/sternfin/mkacperc/public-html/sin.pdf. Consultado en mayo de 2016

Mas, M., Losano, P., Trifaró, N., Owen, M., Fernández, M.V., Ramírez, G.,Krebs, A. (2014). Construcción de un indicador sintético de atractividad (SIET) de un destino turístico. VI Congreso Latinoamericano de Investigación Turística. Neuquén.

Sarmiento Ramírez, Y. (2014). "Evaluación de la competitividad territorial para el proceso de planificación”. Tesis en opción al grado científico de Doctor en Ciencias Económicas. Universidad de Camagüey. Facultad de Ciencias Económicas y Empresariales. Centro de estudios de Dirección empresarial y territorial. Camagüey, Cuba.

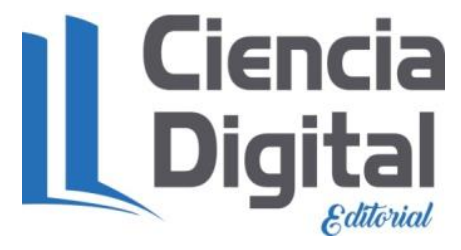


Para citar el artículo indexado.

Medina Labrada, J., Pérez Ricardo, E. del C., \& Riquenes Gainza, M. de las M. (2019). Atractividad - Competitividad de destino turístico. Evaluación de Holguín para el mercado estadounidense. ConcienciaDigital, 2(4), 43-55.

https://doi.org/10.33262/concienciadigital.v2i4.851

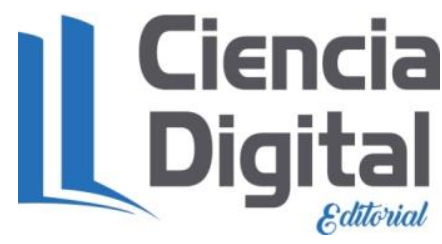

El artículo que se publica es de exclusiva responsabilidad de los autores y no necesariamente reflejan el pensamiento de la Revista Conciencia Digital.

El articulo queda en propiedad de la revista y, por tanto, su publicación parcial y/o total en otro medio tiene que ser autorizado por el director de la Revista Conciencia Digital.
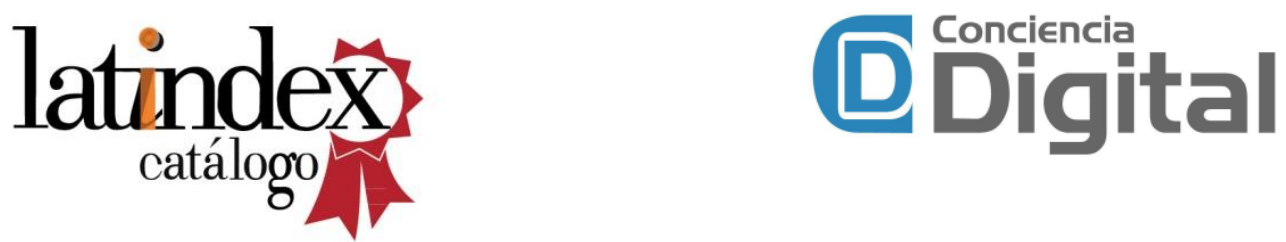